\title{
TU/e EmonOWEN

\section{Mesoscale simulation of soft particles with tunable contact angle in multicomponent fluids}

\section{Citation for published version (APA):}

Wouters, M., Aouane, O., Krüger, T., \& Harting, J. (2019). Mesoscale simulation of soft particles with tunable contact angle in multicomponent fluids. Physical Review E, 100(3), [033309].

https://doi.org/10.1103/PhysRevE.100.033309

DOI:

10.1103/PhysRevE.100.033309

Document status and date:

Published: 17/09/2019

\section{Document Version:}

Publisher's PDF, also known as Version of Record (includes final page, issue and volume numbers)

\section{Please check the document version of this publication:}

- A submitted manuscript is the version of the article upon submission and before peer-review. There can be important differences between the submitted version and the official published version of record. People interested in the research are advised to contact the author for the final version of the publication, or visit the $\mathrm{DOI}$ to the publisher's website.

- The final author version and the galley proof are versions of the publication after peer review.

- The final published version features the final layout of the paper including the volume, issue and page numbers.

Link to publication

\section{General rights}

Copyright and moral rights for the publications made accessible in the public portal are retained by the authors and/or other copyright owners and it is a condition of accessing publications that users recognise and abide by the legal requirements associated with these rights.

- Users may download and print one copy of any publication from the public portal for the purpose of private study or research.

- You may not further distribute the material or use it for any profit-making activity or commercial gain

- You may freely distribute the URL identifying the publication in the public portal.

If the publication is distributed under the terms of Article 25fa of the Dutch Copyright Act, indicated by the "Taverne" license above, please follow below link for the End User Agreement:

www.tue.nl/taverne

Take down policy

If you believe that this document breaches copyright please contact us at:

openaccess@tue.nl

providing details and we will investigate your claim. 


\title{
Mesoscale simulation of soft particles with tunable contact angle in multicomponent fluids
}

\author{
Maarten Wouters, ${ }^{1, *}$ Othmane Aouane, ${ }^{2, \dagger}$ Timm Krüger, ${ }^{3, \ddagger}$ and Jens Harting $\odot^{2,1, \S}$ \\ ${ }^{1}$ Department of Applied Physics, Eindhoven University of Technology, De Rondom 70, 5612 AP, Eindhoven, the Netherlands \\ ${ }^{2}$ Helmholtz Institute Erlangen-Nürnberg for Renewable Energy, Forschungszentrum Jülich, Fürther Strasse 248, Nürnberg, Germany \\ ${ }^{3}$ School of Engineering, Institute for Multiscale Thermofluids, The University of Edinburgh, Edinburgh EH9 3FB, Scotland, United Kingdom
}

(Received 25 April 2019; published 17 September 2019)

\begin{abstract}
Soft particles at fluid interfaces play an important role in many aspects of our daily life, such as the food industry, paints and coatings, and medical applications. Analytical methods are not capable of describing the emergent effects of the complex dynamics of suspensions of many soft particles, whereas experiments typically either only capture bulk properties or require invasive methods. Computational methods are therefore a great tool to complement experimental work. However, an efficient and versatile numerical method is needed to model dense suspensions of many soft particles. In this article we propose a method to simulate soft particles in a multicomponent fluid, both at and near fluid-fluid interfaces, based on the lattice Boltzmann method, and characterize the error stemming from the fluid-structure coupling for the particle equilibrium shape when adsorbed onto a fluid-fluid interface. Furthermore, we characterize the influence of the preferential contact angle of the particle surface and the particle softness on the vertical displacement of the center of mass relative to the fluid interface. Finally, we demonstrate the capability of our model by simulating a soft capsule adsorbing onto a fluid-fluid interface with a shear flow parallel to the interface, and the covering of a droplet suspended in another fluid by soft particles with different wettability.
\end{abstract}

DOI: 10.1103/PhysRevE.100.033309

\section{INTRODUCTION}

In many fields, soft particles at interfaces play an important role, for example, medical applications [1,2], cosmetics [3], food industry [4], and paints and coatings [5,6]. For a single particle one can still analytically describe simple systems. However, when multiple particles interact with each other, it is typically no longer possible to predict the deformation dynamics of the individual particles, especially when fluidfluid or fluid-vapor interfaces are present.

Computational methods can be of great value to complement experiments and to characterize emergent phenomena stemming from the complex particle-particle and particlefluid interactions, such as the margination of platelets in blood flow [7,8], the rheology of a suspension of soft capsules [9-11], or the stabilization of emulsions with (solid) nanoparticles $[12,13]$.

Simulating soft particles at fluid interfaces can be done on the microscopic scale, with for instance molecular dynamics simulations where one resolves the polymer chains or molecules which make up the soft particle [14]. Such methods are, however, extremely costly and therefore limited to simulations of only a few particles. Macroscopic methods typically directly solve the Navier-Stokes equations, or a coupled set of equations, discretized onto a grid to make it numerically accessible, such as for example finite element

\footnotetext{
*m.p.j.wouters@tue.nl

†o.aouane@fz-juelich.de

†timm.krueger@ed.ac.uk

$\S$ j.harting@fz-juelich.de
}

methods. To properly describe fluid-structure interactions, this often requires expensive remeshing steps and thereby becomes increasingly complex to solve, both in implementation and computational effort [15].

Our goal is an efficient method which makes large-scale simulations of suspensions of soft particles feasible on existing high-performance computing clusters, and offers the possibility to easily extend the simulated system to more complex cases. The mesoscopic lattice Boltzmann method (LBM) is a suitable candidate since it is straightforward to implement and has proven its capability of efficiently simulating large systems of suspended particles in a fluid [16-18].

Simulations of soft particles in a single-component fluid are well established in the LBM literature. Typical examples are fluid-filled capsules and cells [9,11,19,20], vesicles [21-23], and particles with three-dimensional elasticity [24,25]. Comparably, there are various approaches to model rigid particles in multicomponent fluids. Several authors apply the momentum exchange method introduced by Ladd [18,26] for the solid particle, coupled to the pseudopotential multicomponent fluid method of Shan and Chen [27-30] or a free-energy-based multicomponent model [31,32]. Other methods for the fluid-structure coupling exist as well, such as fluid particle dynamics [33], or the immersed boundary method $[34,35]$.

Despite the abundance of methods for rigid colloids in multicomponent fluids, the simulation of soft particles in multicomponent fluids is still sparse in the LBM literature. Li et al. [36] coupled the pseudopotential method of Shan and Chen [37,38] with the immersed boundary method of Peskin [39]. Pepona et al. [40] have recently presented a two-dimensional model for soft particles at fluid-fluid 
interfaces based on the free-energy LBM coupled with immersed boundaries. The immersed boundary method interpolates the local velocity around each boundary node via an interpolation stencil in order to acquire the velocity of the boundary node. Overlap of these interpolation stencils is inherent to the model, resulting in a small mass flux through the boundary. Li used an improved scheme which corrects for the overlap of the stencils in order to ensure the no-slip condition at moving solid boundaries [41], and finally applied the method to deformable cilia, which are partially at the interface between two fluids. Their method is, however, costly due to the need of inverting a square matrix of the size of the number of discretization points for the boundary. Since their cilia are discretized with roughly 20 boundary nodes, this does not pose a significant problem for the computational efficiency. However, for systems with many soft particles in which a single particle can typically have of the order of $O\left(10^{3}\right)$ boundary nodes, the impact of this matrix inversion quickly becomes a computational bottleneck.

In this work we propose an alternative approach which is capable of describing soft particles with a tunable contact angle, in three-dimensional multicomponent fluids in large and/or dense systems. It combines several existing methods commonly used in the LBM literature. Our basis is the momentum exchange bounce-back fluid-structure coupling as introduced by Ladd [18,26], coupled to soft particles in a similar manner as introduced for single-component systems by Aidun et al. [11,16,27,42]. This is then coupled to the popular multicomponent pseudopotential method of Shan and Chen (SC) $[37,38]$ and allows for tunable preferential contact angles of the particle surface via an effective offset in the densities used for the force calculations as introduced by Jansen et al. [29].

The presented method has the benefit that the no-mass-flux condition across the particle boundary is guaranteed inherently thanks to the bounce-back conditions. Furthermore, the fluid-structure coupling is independent of the collision step of the LBM and the chosen constitutive models for the soft particles, which ensures a lot of freedom for future extensions or adaptations of the model. Finally, our method allows to tune properties at the level of the used discretization of the boundary, opening the possibility to, for example, simulate soft particles with inhomogeneous wetting properties across the surface.

For clarity, we present the method in the most simple implementation possible and refer to the existing literature for possible extensions and improvements. We use the Skalak (SK) hyperelastic constitutive law [43] to model the fluidfilled capsules throughout this work, but we emphasize that other existing soft particle models $[24,44,45]$ can be applied straightforwardly as well.

The remainder of the paper is structured as follows. In Sec. II we introduce the simulation method and provide brief summaries of the different existing models combined in our approach. We validate our method in Sec. III. First, the deformation of a single capsule in shear flow is compared to previously reported values in the literature. Then, the error in the equilibrium shape of capsules adsorbed onto fluid-fluid interfaces is studied, where we compare our data to a reference model to explicitly exclude all causes of errors other than the used fluid-structure coupling, since the used models for soft particles are already well reported in literature. Also, the influence of a shear flow parallel to the fluid-fluid interface on the steady-state shape of a soft particle is briefly studied. Furthermore, we demonstrate the possibilities of our method by simulating the adsorption of soft capsules with different wettability onto a droplet. Finally, we summarize and discuss our results in Sec. IV.

\section{METHOD}

\section{A. Lattice Boltzmann method}

To simulate the dynamics of the fluids, we apply the lattice Boltzmann method (LBM). It allows for a straightforward implementation and has proven its capability to handle a wide variety of fluid dynamics problems [46-48]. Thanks to the locality of the algorithm, it is also well suited for a parallel implementation and high performance computing applications $[16,17]$.

The LBM is based on the discretization of the Boltzmann equation, where we discretize space into a regular threedimensional (3D) lattice with positions $\mathbf{x}$ and lattice spacing $\Delta x$, and the time $t$ into evenly spaced time steps with step size $\Delta t$. Additionally, we discretize the velocity space with 19 discrete lattice velocities $\mathbf{c}_{i}$ (D3Q19 model). Using the Bhatnagar-Gross-Krook (BGK) collision operator [49], the time evolution of the single-particle probability distribution function $f_{i}$ is described by

$f_{i}\left(\mathbf{x}+\mathbf{c}_{i} \Delta t, t+\Delta t\right)-f_{i}(\mathbf{x}, t)=-\frac{\Delta t}{\tau}\left[f_{i}(\mathbf{x}, t)-f_{i}^{\mathrm{eq}}(\mathbf{x}, t)\right]$,

where $\tau$ is a relaxation time associated with the kinematic viscosity as $\nu=c_{s}^{2}(\tau-0.5) \Delta x^{2} / \Delta t$, and $c_{s}=\sqrt{1 / 3} \Delta x / \Delta t$ is the speed of sound.

The left-hand side of Eq. (1) describes the streaming step during which the distribution functions $f_{i}$ are streamed from fluid node $\mathbf{x}$ along their respective lattice directions $\mathbf{c}_{i}$ to the neighboring fluid node $\mathbf{x}+\mathbf{c}_{i}$. The right-hand side denotes the intermolecular collisions and resulting relaxation toward a local equilibrium distribution with characteristic timescale $\tau$. We use a second-order accurate approximation for the equilibrium distribution $f_{i}^{\mathrm{eq}}$, given as

$$
f_{i}^{\mathrm{eq}}(\mathbf{x}, t)=w_{i} \rho\left[1+\frac{1}{c_{s}^{2}}\left(\mathbf{c}_{i} \cdot \mathbf{u}\right)+\frac{1}{2 c_{s}^{4}}\left(\mathbf{c}_{i} \cdot \mathbf{u}\right)^{2}-\frac{1}{2 c_{s}^{2}} u^{2}\right],
$$

where the lattice weights $w_{i}$ are defined as

$$
w_{i}=\left\{\begin{array}{lll}
1 / 3 & i=0 & \text { (resting) } \\
1 / 18 & i=1 \ldots 6 & \text { (orthogonal) } \\
1 / 36 & i=7 \ldots 18 & \text { (diagonal) }
\end{array}\right.
$$

to ensure isotropy.

We include forces $\mathbf{F}(\mathbf{x})$ acting on the fluid as a shift in the equilibrium velocity in Eq. (2) [37]. From the distribution 
function $f_{i}$ the local macroscopic density $\rho$ and velocity $\mathbf{u}$ can now be computed as

$$
\begin{gathered}
\rho(\mathbf{x})=\rho_{0} \sum_{i} f_{i}(\mathbf{x}), \\
\rho(\mathbf{x}) \mathbf{u}(\mathbf{x})=\rho_{0} \sum_{i} f_{i}(\mathbf{x}) \mathbf{c}_{i}+\frac{\rho_{0} \Delta t}{2} \mathbf{F}(\mathbf{x}),
\end{gathered}
$$

where $\rho_{0}$ is an arbitrary scaling factor for the mass density of the fluid, which is fixed to unity throughout this work. For more details and an extensive derivation of the LBM we refer the interested reader to $[47,48]$.

\section{B. Soft particle model}

We consider a fluid-filled capsule modeled as a twodimensional (2D) incompressible hyperelastic membrane discretized into regular triangular finite elements. The membrane mechanical properties depend on the chosen hyperelastic law. We use in this work the Skalak constitutive model which provides the membrane with resistance to local area dilatation and shear elasticity [43]. The corresponding strain energy reads as

$$
E^{\text {strain }}=\frac{\kappa_{S}}{4} \oint\left(I_{1}^{2}+2 I_{1}-2 I_{2}+C I_{2}^{2}\right) d A, \quad C>-1 / 2
$$

where $\kappa_{S}$ is the shear elastic modulus, $I_{1}=\lambda_{1}^{2}+\lambda_{2}^{2}-2$ and $I_{2}=\lambda_{1}^{2} \lambda_{2}^{2}-1$ are the strain invariants of the deformation tensor, and $\lambda_{1}$ and $\lambda_{2}$ are the principal stretching ratios. $C$ is a parameter controlling the local inextensibility of the membrane and is related to the area dilation modulus by $\kappa_{A}=$ $(1+2 C) \kappa_{S}$. In the asymptotic limit of small deformation, the 2D Poisson ratio can be expressed as function of $C$ such as $v_{s}=C /(1+C)$ with $\left.\left.v_{s} \in\right]-1 \ldots 1\right]$ [44]. A constraint on the volume variation is enforced using a penalty function given by

$$
E^{\text {volume }}=\frac{\kappa_{V}}{2} \frac{\left(V-V^{0}\right)^{2}}{V^{0}},
$$

with $\kappa_{V}$ a volume-change resistance modulus, and $V^{0}$ the reference volume of the stress-free particle. The strain and volume forces are computed from their respective energy terms via the principle of virtual work [50,51], where the force for each individual boundary node $\mathbf{x}_{b}$ is computed using

$$
\mathbf{F}_{b}=-\frac{\partial E\left(\mathbf{x}_{b}\right)}{\partial \mathbf{x}_{b}} .
$$

The membrane can be endowed with a bending resistance to out of plane deformation by means of the Helfrich free energy [52] given by

$$
E^{\text {bending }}=\frac{\kappa_{B}}{2} \oint\left(2 H-H_{0}\right)^{2} d A,
$$

where $\kappa_{B}$ is the bending modulus, $H$ and $H_{0}$ are the mean and spontaneous curvatures, respectively. For the sake of simplicity, the spontaneous curvature is neglected in this study. The bending surface force density is obtained from the functional derivative of Eq. (9) and reads as

$$
\mathbf{f}_{B}=2 \kappa_{B}\left[2 H\left(H^{2}-K\right)+\Delta_{s} H\right] \mathbf{n},
$$

where $K$ is the Gaussian curvature, $\Delta_{s}$ is the Laplace-Beltrami operator, and $\mathbf{n}$ is the normal vector pointing outward from the particle surface. The nodal bending force is obtained by multiplying Eq. (10) with the corresponding nodal area (Voronoi area). $H, K$, and $\Delta_{s}$ are computed using the discrete differential-geometry operators approach as detailed in Meyer et al. [53]. We define the dimensionless bending parameter $B=\kappa_{B} /\left(\kappa_{S} R_{0}^{2}\right)$, and fix it throughout this work to $B=10^{-2}$ unless specified otherwise.

We apply a first-order accurate forward Euler scheme for the movement of the boundary nodes, to prevent the costly need to recalculate all the forces acting on the boundary nodes at a higher rate than the LBM updates, as it would be required by substep integration techniques. Higher-order time-integration schemes can be used as well, but were found unnecessary for the presented work.

The proposed simulation method of this work can easily be applied to different types of soft particles, such as fluid-filled elastic capsules [44,54], vesicles [45], or soft elastic particles with 3D elasticity [24]. For the latter 3D elastic particles one actually introduces a cross-linked lattice of boundary nodes, rather than a $2 \mathrm{D}$ membrane.

\section{Fluid-structure coupling}

Exact boundary conditions, which prevent any mass flux through the particle surface, are desirable for a correct physical model. For single-component fluids, a small mass flux is, however, typically mitigated by the pressure, where the results can be considered tolerable based on the required accuracy. For a multicomponent fluid the requirement of exact boundary conditions is more important since the interior and exterior fluids of the particle typically have a different composition, where the mass flux through its surface could, for example, trigger the formation of droplets. Hence, we ensure that no mass moves through the surface by applying bounce-back boundary conditions for distribution functions $f_{i}$ that cross a boundary element during the streaming step. We use the halfway bounce-back scheme (BB), as already used for the simulation of soft particle suspensions in single-component fluids [11,42,55], which bounces the incoming distribution functions from a fluid node $\mathbf{x}_{f}$ onto a boundary element back during the streaming step as [26]

$$
f_{j}\left(\mathbf{x}_{f}, t+\Delta t\right)=f_{i}^{*}\left(\mathbf{x}_{f}, t\right)+2 \rho w_{i} \mathbf{c}_{i} \cdot \mathbf{v}_{0} / c_{s}^{2},
$$

where the superscript ${ }^{*}$ indicates the post-collision state, $\mathbf{c}_{i}=$ $-\mathbf{c}_{j}, \rho$ is taken as the local density at fluid node $\mathbf{x}_{f}$, and $\mathbf{v}_{0}$ is the velocity of the boundary element.

The momentum transfer onto the boundary is given by the difference of the incoming and outgoing populations as

$$
\Delta \mathbf{p}_{i}=\Delta x^{3}\left[f_{i}^{*}\left(\mathbf{x}_{f}, t\right)+f_{j}\left(\mathbf{x}_{f}, t+\Delta t\right)\right] \mathbf{c}_{i} .
$$

In the presented work, the coupling of quantities between the boundary elements and boundary nodes is done via a homogeneous scheme where the three nodes of only the corresponding boundary element are given the same weighting factor. This technique maintains the locality of the algorithm by eliminating the need to communicate properties of the boundary node of the neighboring boundary elements. Alternatively, both Buxton et al. [56] and MacMeccan et al. [11] 
introduced different weighting schemes for the spreading of the exchanged momentum to boundary nodes for all nodes within a characteristic distance. However, no differences were observed on the timescale of equilibration for all presented simulations. Hence, for the sake of brevity, a thorough characterization of this free parameter is left for future work. Also, the interplay with the error stemming from the halfway bounce-back as compared to more accurate methods should be included in such a study.

The halfway bounce-back method as given in Eq. (11) is known to suffer from staggered momenta [26,57,58]. Especially in small enclosed volumes, such as our fluid-filled capsule, these momenta rapidly manifest themselves as a staggering in the velocity field. In this work we prevent this by spreading the total exchanged momentum from the halfway bounce-back coupling homogeneously over two consecutive time steps [26].

The staggered momenta can also be prevented by using full-way bounce-back [59], where the boundary is still approximately halfway between two fluid nodes, but the populations $f_{i}$ travel the complete distance between two fluid nodes and are reversed during the next collision step. However, for the full-way bounce-back one needs to store the populations that pass a boundary element, which is impracticable when there are fluid nodes on both sides of the boundary. Alternatively, one could also increase the accuracy of the discretization of the boundary with interpolated bounce-back boundary conditions [60], multireflection boundary conditions [61], or the one-point second-order bounce-back boundary condition [62]. They do offer a better representation of the boundary on the fluid lattice, and remove the force fluctuations that stem from the jump that the location of the discretized boundary makes when a boundary element moves past a fluid node in the halfway bounce-back algorithm. However, they are computationally more expensive since one needs to calculate the exact distance between a fluid node and the boundary element along the lattice vectors. Furthermore, the interpolated bounce-back and multireflection boundary conditions use information of multiple fluid nodes, which increases the minimum separation between two adjacent particles. Hence, the halfway bounce-back algorithm with the coupling proposed by Ladd [26] and a first-order accurate time-integration scheme is deemed most effective for applications involving large systems, where the particles could become densely packed.

\section{Multicomponent fluid interactions}

To describe multicomponent fluids, we use the pseudopotential method of Shan and Chen $[37,38]$. For each component $c$ we individually solve Eq. (1). In order to couple the different components, a repulsive fluid interaction force is added between opposing fluid components $c$ and $c^{\prime}$ at neighboring fluid nodes, as given by

$$
\mathbf{F}^{\mathrm{SC}}=-\psi^{c}\left(\mathbf{x}_{f}, t\right) \sum_{c^{\prime}} G^{c c^{\prime}} \sum_{i} w_{i} \psi^{c^{\prime}}\left(\mathbf{x}_{f}+\mathbf{c}_{i}, t\right) \mathbf{c}_{i}
$$

where $\psi^{c}$ is a pseudopotential, and $G^{c c^{\prime}}$ is the fluid interaction strength. For this work we chose $\psi^{c}=1-\exp \left(-\rho^{c} / \rho_{0}\right)$ and limit the number of components to 2 .

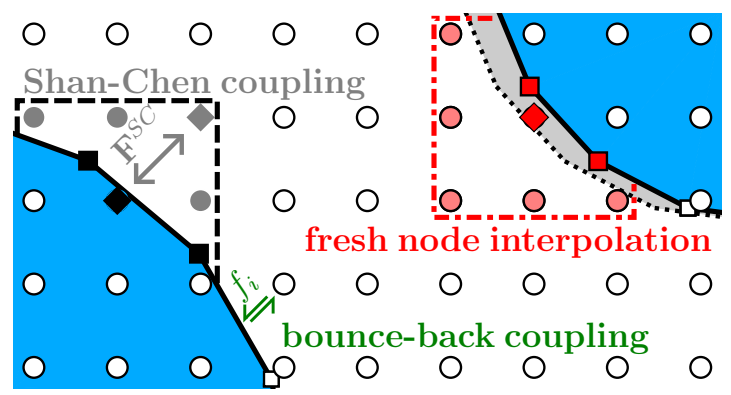

FIG. 1. Schematic, two-dimensional visualization of the fluidstructure coupling with the lattice of fluid nodes (circles+diamonds). The soft particles (light blue areas) are represented as an infinitely thin membrane consisting of $N_{b}$ boundary nodes (squares) and $N_{f}$ boundary elements (solid lines). The fluid encapsulated by the boundaries is decoupled from the bulk region by bounce-back boundary conditions (dark green arrows). In order to calculate fluid interaction force $F^{\mathrm{SC}}$ between fluid nodes on opposite sides of the boundary (gray and black diamonds), we interpolate the fluid density on the opposing side of the boundary. For the black diamond node, this corresponds to the gray nodes in the dashed box. The fluid interaction force that would be applied at the interpolated node is distributed over the boundary nodes that span the face over which the force would act (solid black squares). When the boundary moves, a fluid node can become uncovered by the particle (red triangular node in gray shaded region). In such a case, we interpolate the new fluid density from the nearest neighboring nodes (red circles inside the dotted box), where we ensure momentum conservation by adding the removed momentum and subtracting the created momentum to the boundary nodes (solid red squares) of the boundary element that crossed over the node.

The interior and exterior fluids are decoupled at the boundary via the bounce-back boundary conditions. Therefore, we also decouple the interior and exterior fluid interaction forces. In order to satisfy continuity close to the boundary, we interpolate the neighboring fluid densities located on the opposing side of a boundary

$$
\rho_{\text {bound }}^{c}\left(\mathbf{x}_{f}, t\right)=\frac{1}{N_{i}^{\text {opp }}} \sum_{i} \rho^{c}\left(\mathbf{x}_{f}+\mathbf{c}_{i}, t\right),
$$

where $i$ runs over all $N_{i}^{\text {opp }}$ fluid nodes on the opposite side of the boundary as $\mathbf{x}_{f}$, as schematically indicated in Fig. 1 . This way we interpolate the densities for the ring of fluid nodes just outside our boundary for the inner fluid, and the ring of fluid nodes just inside the boundary when calculating the fluid interaction force on the fluid nodes just outside the boundary. In order to conserve momentum, the resulting force that would act on the fluid node across the boundary is applied to the boundary element that separates the set of fluid nodes.

This also allows to tune the contact angle of the particle surface by adding an offset $\rho_{\text {virtual }}^{c}$ to one component of the interpolated densities $\rho_{\text {bound }}^{c}$ of Eq. (14), as is proposed by Jansen et al. [29]. Since the density offset is only added to one of the two components, while the nonpreferred fluid component is unchanged, we define the dimensionless particle color $\Delta \rho$ by normalizing the added virtual density offset 


$$
\rho_{\text {virtual }}^{c} \text { as }
$$

$$
\Delta \rho=\frac{1}{2} \frac{\rho_{\text {virtual }}}{\rho_{\text {maj }}-\rho_{\text {min }}},
$$

where $\rho_{\text {maj }}^{c}$ and $\rho_{\text {min }}^{c}$ are the equilibrium majority and minority densities corresponding to the chosen initial densities in the system and the fluid interaction parameter $G^{c c^{\prime}}$. Here, a positive particle color indicates that the fluid density is added to the first fluid, whereas a negative particle color indicates the density added to the other fluid.

The benefit of decoupling the interior and exterior regions by use of the bounce-back boundary conditions is that we do not need a third fluid component for the interior region of the particles. First, this will save a significant amount of computational resources. Second, the interior region of the particle can be given the same composition as the exterior region, which makes it easier to initialize systems where the hydrostatic pressure inside the particle equals that outside the particle for an undeformed mesh. In this work the interior of the particle is always initialized with the same fluid composition and fluid interaction strength as the exterior region when no fluid-fluid interfaces are present. When fluid-fluid interfaces are present, the composition equals that of the lower fluid layer. Careful checks validated that changing the interior composition to that of the upper fluid yields no difference in the presented results.

\section{E. Fresh fluid nodes}

One of the problems arising for moving boundaries is how to initialize fluid nodes when a boundary element moves over it, commonly referred to as fresh node treatment. For solid particles one can choose not to remove the fluid that becomes covered by a particle [26], however, for fluid-filled capsules this is not possible anymore. In this case, the local fluid at a fresh fluid node $\mathbf{x}_{\text {fresh }}$ needs to be removed and replaced by an appropriate representation of the fluid present on the other side of the boundary, whenever a boundary element passes a fluid node.

Several methods are possible for this, of which we use an interpolation method $[29,63]$. The new density at the fresh fluid node is given by

$$
\rho_{\text {fresh }}^{c}\left(\mathbf{x}_{\text {fresh }}, t\right)=\frac{1}{N_{i}^{\text {same }}} \sum_{i} \rho^{c}\left(\mathbf{x}_{\text {fresh }}+\mathbf{c}_{i}, t\right),
$$

where the sum runs over all $N_{i}^{\text {same }}$ neighboring fluid nodes that are on the same side of the boundary as the fresh node, which are not fresh nodes themselves during that time step. This interpolation is schematically visualized in Fig. 1. As in Sec. II C, the fresh fluid node is assigned the average velocity of the three boundary nodes $\mathbf{x}_{b}$ of the nearest boundary element

$$
\mathbf{v}\left(\mathbf{x}_{\text {fresh }}, t\right)=\frac{1}{3} \sum_{b=1}^{3} \mathbf{v}_{b}(t) .
$$

In order to locally conserve the combined momentum of the fluids and particles, the momentum of the removed fluid at the fresh node is added to the boundary element that moved over the node, while the momentum of the newly generated fluid is subtracted from the same element, similar as to the

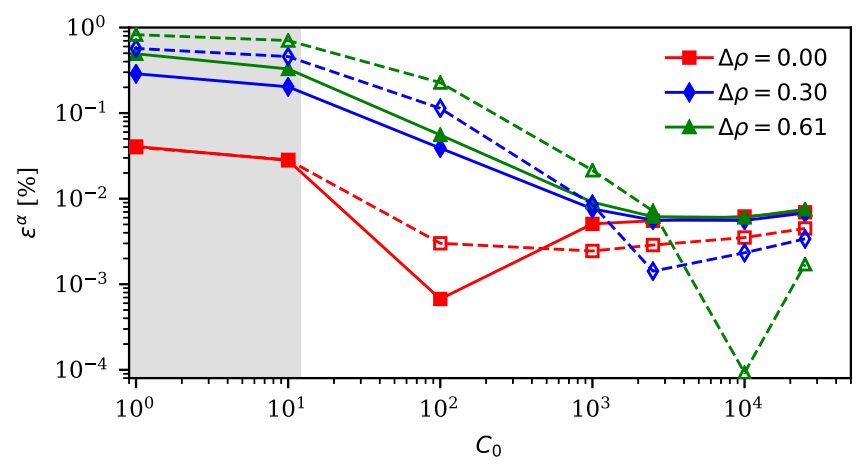

FIG. 2. Relative errors in total mass for different wetting properties of the particle surface and different values of the mass-correction scheme of Eq. (18). A rigid spherical boundary with radius $10 \Delta x$ is initialized at a flat interface between two immiscible components, with a constant velocity of $v_{x}=10^{-3}$ parallel to the interface imposed on each individual boundary node, with a system size of (180, $60,60)$ fluid nodes. The data are sampled after 250000 simulation steps, i.e., on an integer number for the particle displacement to avoid capturing errors due to a change in discretization of the particle on the fluid lattice. The open symbols indicate the relative error for the preferred component, while the solid symbols indicate the relative error for the nonpreferred component. The lines are drawn as a guide to the eye. The region with the gray background indicates the simulations for which the error is still diverging after 250000 simulation steps.

momentum exchange method for solid objects as introduced by Ladd [18].

The interpolation as shown in Eq. (16), however, fails to conserve the global mass in the system when there are forces or density gradients close to the boundary, which is always the case when the particle is near a fluid-fluid interface [12,29]. Close to the particle surface the fluid interaction force results in a slightly lower density, and therefore the interpolation will always slightly over-estimate the local density. When the particle is close to the fluid interface, or has a preferential contact angle other than $90^{\circ}$, this effect increases significantly. Since no analytical solution is readily available for the density profile around the surface, a correction which only uses local quantities is not feasible. Therefore, we apply an adaptive mass-correction term which scales the density of fresh fluid nodes as [12,29]

$$
\rho_{\text {fresh }}^{c}=\left\langle\rho^{c}\right\rangle\left(1-C_{0} \frac{\sum_{c} \rho_{\text {init }}^{c}}{\rho_{\text {init }}^{c}} \frac{\Delta \rho^{c}}{V_{\text {sys }}}\right),
$$

where $V_{\text {sys }}$ is the total volume of all fluid nodes in the system, $C_{0}$ is a parameter used to tune the strength of the adaptive correction, $\rho_{\text {init }}^{c}$ is the average initial density of component $c$ in the entire system, and $\left\langle\rho^{c}\right\rangle$ is the averaged density obtained by Eq. (16). We limit $\rho_{\text {fresh }}^{c}$ between the smallest and largest density value of its neighbors [12].

In Fig. 2 we show the error in the total mass after 250000 simulation steps for a system where a perfectly rigid particle adsorbed onto a fluid-fluid interface is propagated parallel to this interface. The particle movement is imposed by fixing the velocity of each boundary node to $10^{-3}$. Independent of the wetting properties of the particle, the relative error of 
each individual component is below $0.01 \%$ when $C_{0} \geqslant 2500$. Hence, $C_{0}=2500$ is used throughout this work independent of the used particle color.

\section{RESULTS}

Throughout this entire section we fix the characteristic timescale $\tau$, the reference density $\rho_{0}$, and the discretized timescales and length scales $\Delta t$ and $\Delta x$ to unity. However, for clarity purposes we will denote the nondimensionalized lengths and times in units of $\Delta x$ and $\Delta t$. Unless specified otherwise, the fluid interaction strength is set as $G^{c c^{\prime}}=3.6$, and the initial majority and minority densities are set as $\rho_{\text {maj }}^{\text {init }}=0.7$ and $\rho_{\text {min }}^{\text {init }}=0.042$, which yields a fluid-fluid surface tension of $\gamma^{c c^{\prime}}=0.0388$. The particles are discretized with $N_{f}=2880$ boundary elements, and $N_{b}=1442$ boundary nodes, where each boundary node is assigned a mass of $5 \rho_{0} \Delta x^{3}$. The fluid encapsulated by the particle is initialized with the same composition and fluid interaction strength as the lower fluid.

\section{A. Soft capsule in shear flow}

As a first validation of the fluid-structure coupling we simulate the deformation of a capsule in a linear shear flow. We define the capillary number $\mathrm{Ca}$ as

$$
\mathrm{Ca}=\frac{\rho^{\mathrm{tot}} v \dot{\gamma} R_{0}}{\kappa_{S}},
$$

where $\rho^{\text {tot }}$ is the summed fluid density of both components, and $\dot{\gamma}$ is the shear rate. We initialize a single particle with a radius $R_{0}=7.64 \Delta x$, a shear elasticity $\kappa_{S}$, and local extensibility $C=1$ (i.e., $\kappa_{B}=0$ and $B=0$ ) at the center of a cubic domain of $48^{3}$ fluid nodes with a homogeneous binary fluid mixture of $\rho^{c}=\rho^{c^{\prime}}=0.5$. The interaction strength is set to $G^{c c^{\prime}}=0.0$, mimicking a homogeneous mixture of noninteracting fluids. A constant shear rate is imposed at the top and bottom $x y$ planes via onsite velocity boundary conditions $[64,65]$. The capillary number is varied by changing the shear elasticity while keeping the shear rate constant at $\dot{\gamma}=10^{-4}$.

For different shear rates, we measure the steady-state Taylor deformation parameter

$$
D=\frac{r_{\text {long }}-r_{\text {short }}}{r_{\text {long }}+r_{\text {short }}}
$$

where $r_{\text {long }}$ and $r_{\text {short }}$ are the length of the long and short halfaxis, respectively. The lengths of these axes are obtained from the tensor of inertia of the boundary surface [66]. As shown in Fig. 3, our simulations show an excellent agreement with the results reported by Lac et al. [67] and Bagchi et al. [68] over a wide range of capillary numbers. As expected, this indicates that in the absence of a fluid interaction force we obtain the correct physics when switching from a single-component fluid to a multicomponent fluid.

\section{B. Adsorption onto a fluid-fluid interface}

In this section we show the capability of our method to model the adsorption of soft particles at fluid-fluid interfaces, and aim to characterize the error stemming from the coupling between the multicomponent fluid and the bounce-back

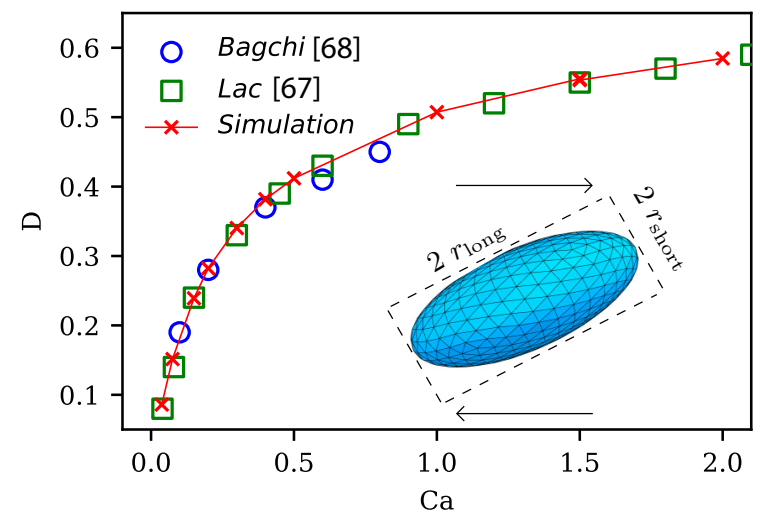

FIG. 3. Taylor deformation parameter $D$ for a soft capsule with local membrane in-extensibility $C=1$, homogeneous fluid densities $\rho^{c}=\rho^{c^{\prime}}=0.5$, and interaction parameter $G^{c c^{\prime}}=0.0$ for different capillary numbers $\mathrm{Ca}$. Our simulations show an excellent agreement with results reported previously in literature $[44,67,68]$. The inset visualizes the steady-state shape for $\mathrm{Ca}=0.5$, where the dashed box indicates the short and long axis, and the arrows indicate the direction of the shear flow.

boundary conditions for soft particles. Soft particles adsorb onto nearby fluid-fluid interfaces in order to minimize the interfacial energy. For rigid particles, the interplay between the inertia of the particle and the surface tension of the fluid-fluid interface dominates the adsorption dynamics. Soft particles have another degree of freedom, namely, the softness of the particle.

All simulations in this section are performed in a system with a fluid-fluid interface in the $x y$ plane, located at $z_{\text {interface }}=$ $32 \Delta x$ in a cubic domain of $(64 \Delta x)^{3}$ fluid nodes. The system is closed at the top and bottom with rigid boundaries using the halfway bounce-back scheme, and periodic boundary conditions in the other directions.

We define the dimensionless softness parameter of the capsule at a fluid-fluid interface as $\beta=R_{0}^{2} \gamma^{c c^{\prime}} / \kappa_{B}$. Unless specified otherwise, we initialize the particles with an initially spherical shape with $R_{0}=10 \Delta x$ and $N_{f}=2880$ boundary elements. The volume conservation modulus is chosen on purpose at a large value of $\kappa_{V}=20$ to ensure a quasi-volumeincompressible capsule with fluctuations in the encapsulated volume in the order of $0.01 \%$ for all presented simulations. The exact choice here is, however, not significant for the presented work, and smaller moduli are possible as well.

In Fig. 4 we visualize the adsorption of a soft particle with $\beta=100$ and 1000 onto a fluid-fluid interface, compared against a rigid particle. For now we focus qualitatively on the dynamics of an adsorbing particle, and therefore only visualize the first $1600 \Delta t$ time steps, in which the particle adsorbs onto the fluid-fluid interface and still shows a small, dampened movement around its final equilibrium position.

As expected from a physical viewpoint, we observe for all three cases a simultaneous downward motion of the interface toward the particle surface, while the top of the particle extends at the fluid-fluid interface under the outward pull of the surface tension. Increasing the softness parameter appears to mitigate the downward movement of the interface, where the height difference of the fluid-fluid interface at the contact 


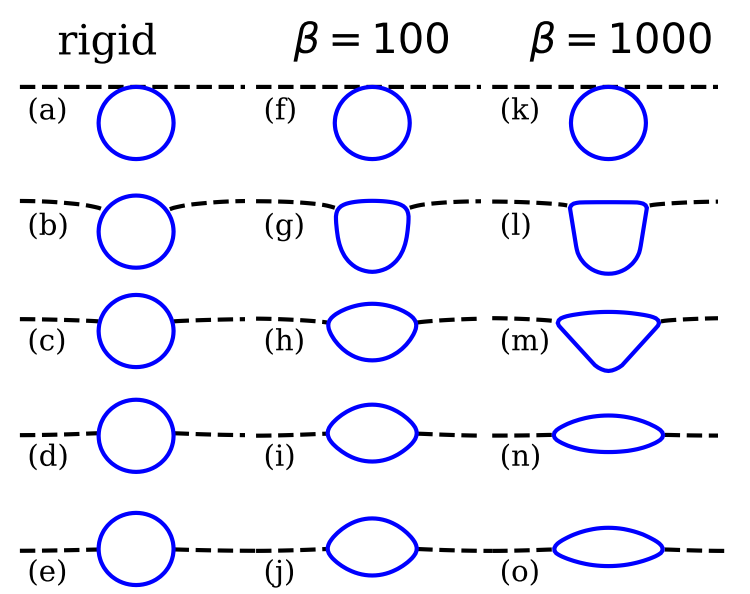

FIG. 4. Visualization of adsorption of a particle (blue solid line) onto a fluid-fluid interface (black dashed line), on a slice perpendicular to the fluid-fluid interface centered at the particle center. The system is initialized with the top of the particle touching an undisturbed fluid-fluid interface. The top panels show the initial state at $t=0$, and each consecutive frame below shows the systems $400 \Delta t$ later. (a) -(e) Adsorption of a perfectly rigid particle. (f)-(j) Adsorption of a particle with softness parameter $\beta=100$. (k)-(o) Adsorption of particle with $\beta=1000$. Due to the inertia of the particle boundary, the fluid-fluid interface can be observed to be pulled downward in the second and third rows of panels, whereas it is slightly pulled upward in the fourth and fifth rows.

point and at the point farthest away from the particle is $2.3 \Delta x$ for the rigid particle, $1.8 \Delta x$ and $1.1 \Delta x$, respectively, for the particle with $\beta=100$ and 1000 . This can be qualitatively explained by the fact that for very soft particles it is energetically more favorable to match the contact angle between the particle surface and fluid-fluid interface via deformation of the surface than via a deformation of the interface. It has to be noticed that the system-size here is relatively small to study such deformation effects. The capillary length can be multiple times larger than the particle radius, which requires larger system sizes. A more thorough and quantitative study of such effects is left for future work, and beyond the scope of this paper.

Many different studies have already been performed on the accuracy of various constitutive models for the dynamics of soft particles. For instance, Ramanujan studied the deformation of capsules for various fluid viscosities [66], Omori studied the correlation between spring network models and continuum constitutive laws [69], Barthès-Biesel extensively studied the deformation of various types of capsules [44], and the discretization of bending algorithms was studied by both Guckenberger [70] and Tsubota [71]. Hence, we purposefully attempt to isolate the error due to the fluid-structure coupling, which is what is central to the proposed method in this work, from those stemming from the method used to model the elastic boundaries.

For this purpose we design a reference model that uses the same routines as our implemented code as presented in Sec. II, but disables the hydrodynamics by ignoring all routines related to the LBM. Instead of the hydrodynamic force of LBM, we directly apply the tensile force $F^{\text {tensile }}$ of the surface tension acting on the particle surface on all boundary

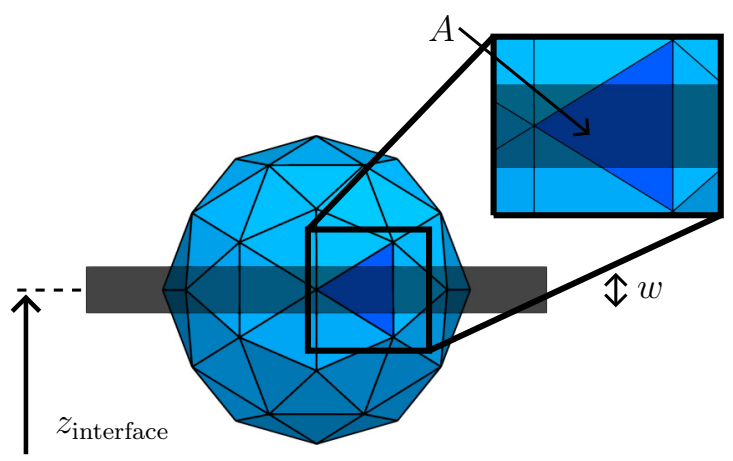

FIG. 5. Schematic visualization for determining the emulated tensile force from the fluid-fluid surface tension acting on a boundary element which is sliced by the interface position in the used reference model. The interface is assumed to have a width $w$ around the interface position $z_{\text {interface }}$ (gray box). We divide the desired surface tension based on the overlapping area $A$ of the boundary element (dark blue) and interface region, and divide the force evenly over the three boundary nodes that span the triangular boundary element.

elements within a width $w$ of a stationary emulated position of the interface. The emulated tensile force on a boundary element is computed from the area of the boundary element covered by the emulated interface as

$$
F^{\text {tensile }}=\frac{\gamma A}{w}
$$

where $A$ is the area of the boundary element which lies between two planes within a distance $w / 2$ of the emulated interface position $z_{\text {interface}}$, and $\gamma$ is the fluid-fluid surface tension. The determination of the area $A$ is schematically visualized in Fig. 5. The emulated tensile force is distributed homogeneously over the three boundary nodes which span the boundary element. We set the width $w=1 \Delta x$, equal to the characteristic length scale of the fluid lattice, unless specified otherwise.

The reference model uses exactly the same elastic laws, the same discretization of the particle surface, and the boundary nodes are updated with the same time-integration scheme. The input values for the surface tension are obtained from separate simulations mimicking a Laplace test, which relate the pressure difference of a droplet for a specific interaction parameter $G^{c c^{\prime}}$ with the droplet radius (data not shown) [12]. This reference model a priori excludes sources of errors other than the fluid-structure coupling, and allows us to compare the simulation results directly against the deformation of any constitutive elastic law already established in the literature.

To quantify the error of our results, we use the extrema of the particle at the poles $r_{p}$ and at the fluid-fluid interface $r_{i}$, where $r_{p}$ is the equilibrium half-axis in the plane perpendicular to the fluid-fluid interface, and $r_{i}$ the equilibrium half-axis in the plane parallel to the fluid-fluid interface. The errors are then defined as the normalized difference between the reference case and our simulation method as

$$
\begin{aligned}
\Delta_{p} & =\frac{\left\langle r_{p}^{\mathrm{ref}}\right\rangle-\left\langle r_{p}^{\mathrm{sim}}\right\rangle}{R_{0}}, \\
\Delta_{i} & =\frac{\left\langle r_{i}^{\mathrm{ref}}\right\rangle-\left\langle r_{i}^{\mathrm{sim}}\right\rangle}{R_{0}}
\end{aligned}
$$


(a)
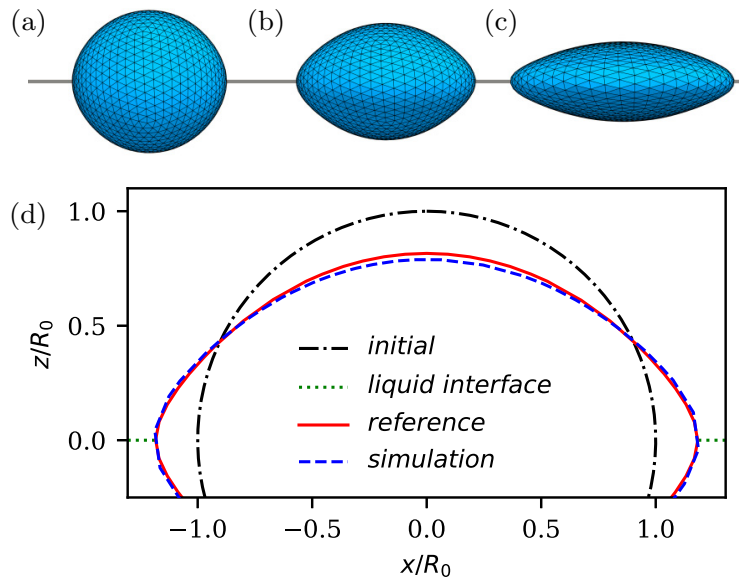

FIG. 6. Equilibrium shapes of a soft capsule adsorbed onto a fluid-fluid interface. (a)-(c) The equilibrium shape for a capsule with $v_{s}=0.9$ and softness parameter of, respectively, $\beta=$ $[10,100,1000]$. The fluid-fluid interface (not shown) runs horizontal through the center of the particles. (d) Overlap of equilibrium shape of our numerical method (dashed-dotted line) and the shape obtained with the reference model of Eq. (21) (solid line) for $\beta=100$ and $v_{s}=0.9$. The dotted line shows the initial, stress-free shape of the capsule, and the green shaded area indicates the lower fluid component.

since due to the bending rigidity the entire shape is characterized by these extrema in equilibrium. The extrema $r_{p}$ and $r_{i}$ can be obtained directly from the tensor of inertia, similar as done for Taylor deformation parameter $D$ of Fig. 3. In this manner, the errors $\Delta_{p}$ and $\Delta_{i}$ provide an intuitive dimensionless measure for the error stemming from the fluidstructure coupling.

In Fig. 6, we visualize the obtained equilibrium shapes for a Poisson ratio $v_{s}=0.9$ and softness parameters $\beta$ of 10, 100, and 1000 to elucidate the used parameters. Furthermore, we visualize a comparison of the obtained equilibrium shape between our simulation method and the reference simulation for $\beta=1000$. The particle is stretched to $r_{i}^{\text {sim }}=11.55 \Delta x$ and $r_{p}^{\text {sim }}=7.49 \Delta x$, with corresponding errors of $\Delta_{p}=-2.2 \times$ $10^{-2}$ and $\Delta_{i}=1.6 \times 10^{-2}$.

Over a wide range of softness parameters $\beta$ and Poisson ratios $v_{s}$ we observe an excellent agreement with the reference model, as shown in Fig. 7. For $\beta=1000$ and $v_{s}=0.9$ the ratio between the axis in the plane perpendicular and parallel to the fluid-fluid interface is 2.3 , with an absolute mismatch at the fluid-fluid interface of only $\left\langle r_{i}^{\mathrm{ref}}\right\rangle-\left\langle r_{i}^{\mathrm{sim}}\right\rangle=0.31 \Delta x$, which is considered as excellent agreement when considering the accuracy of the used halfway bounce-back boundary conditions.

The increasing error for larger softness parameters can be understood by considering the minor axis of the particle perpendicular to the fluid-fluid interface, which decreases for an increased softness and approaches the diffuse interface width $\xi$ of the multicomponent model for very large softness parameters. The largest deformation is observed for a particle with $\beta=1259$ and $\nu_{s}=0.75$, where the minor axis decreases down to $3.6 \Delta x$ and the errors with an emulated width of 1 read as $\Delta_{p}=-3.3 \times 10^{-2}$ and $\Delta_{p}=6.3 \times 10^{-2}$ at the
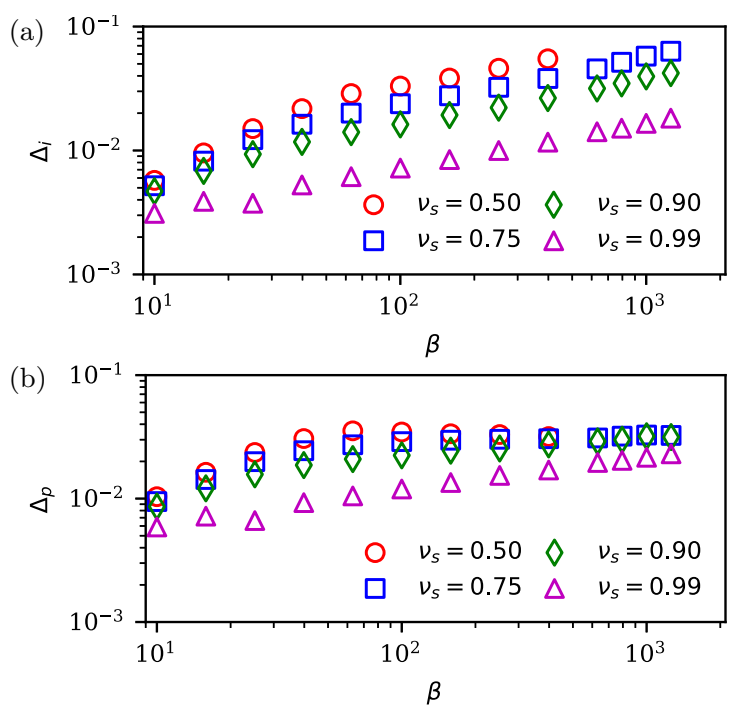

FIG. 7. Characterization of the error at (a) the interface $\Delta_{i}$ and (b) poles $\Delta_{p}$ in the equilibrium shapes as defined in Eq. (22) for different softness parameters $\beta$ and Poisson ratio $v_{s}$ for a particle with radius $R_{0}=10 \Delta x$.

poles and interface, respectively. The typical width of the diffuse interface for the used interaction parameter $G^{c c^{\prime}}=3.6$ is $\xi \simeq 4 \Delta x$. When we increase the emulated width $w$ of the interface, we indeed observe a decrease in the error at both the poles and the interface, where for an emulated width of $w=4$ the errors reduce to $\Delta_{p}=-2.2 \times 10^{-2}$ and $\Delta_{p}=$ $3.3 \times 10^{-2}$. As can be seen in Fig. 8 the errors for particles with a very large initial radius are only weakly dependent on the particle radius and appear to approach a uniform value independent of the emulated interface width when $R_{0} \gg \xi$. For smaller intermediate particle radii $R_{0} \lesssim \xi$ the importance

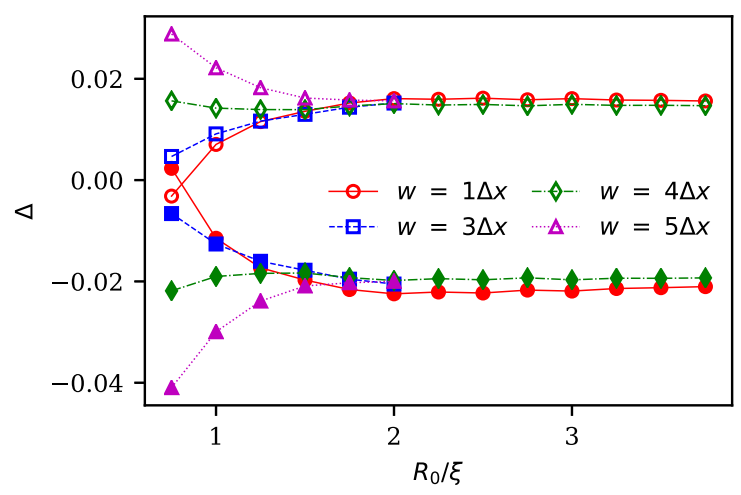

FIG. 8. Characterization of the errors in the equilibrium shapes as defined in Eq. (22), for a capsule with fixed softness parameter $\beta=100$ and Poisson ratio $v_{s}=0.9$ for different initial radii $R_{0}$ and emulated interface width $w$. The open symbols indicate the error at the fluid-fluid interface $\Delta_{i}$, and the solid symbols the error at the poles $\Delta_{p}$. For small radii we clearly observe the influence of the diffuse interface stemming from the pseudopotential LBM, whereas we achieve a roughly constant error for different radii when using an interface width of $w=4 \Delta x$ for the reference model. The lines for $w=3 \Delta x$ and $5 \Delta x$ are cropped after $R_{0} / \xi=2$ for clarity since they closely follow the line for $w=4 \Delta x$ in this regime. 
of the diffuse interface becomes apparent, where the errors with a very low emulated width $w$ become strongly dependent on the ratio of the particle radius and diffuse interface width.

To verify the Galilean invariance of the presented method, a soft particle with $\beta=500$ and $v_{s}=0.9$ adsorped onto a fluid-fluid interface is studied where the top and bottom plane of the system are driven with a constant velocity $\mathbf{v}=$ $[0.001,0.002,0] \Delta x / \Delta t$ parallel to the interface. In order to exclude effects from the initial acceleration of the particle, the simulation is run for 500000 simulation steps, of which the last 50000 are sampled every 5000 steps. No observable differences were found in the particle shape, and the pressure inside and around the particle only shows a relative difference of $0.03 \%$. Due to the pseudopotential multicomponent fluid method, spurious currents are present near the contact point of the particle boundary with the fluid-fluid interface, similar to the spurious currents that are known to persist indefinitely near the curved fluid-fluid interfaces. Small differences occur in the fluid velocities around the contact point on the timescale of $\Delta t$ as a result of the continuously changing discretization of the particle boundary on the fluid lattice. These stem from the halfway bounce-back scheme, which is known to induce local force fluctuations whenever a boundary element jumps over a fluid node. In Fig. 9 we compare the magnitude of the spurious velocities for a stationary particle and a particle moving along with the system with velocity $\mathbf{v}$. Here, it can be observed that the spurious currents do not show an increase in magnitude, but do show a minor increase in their extent. However, for this work we deem the accuracy of the halfway bounce-back scheme sufficient. In future works, when a higher accuracy is required, the errors stemming from the changing discretization of the particle boundary on the fluid lattice could be mitigated by using a fluid-structure coupling scheme with a higher accuracy. Similarly, the effect of the spurious currents could be reduced by exchanging the multicomponent model for a scheme with reduced spurious currents.

\section{Soft capsule at an interface under shear}

In this section we briefly demonstrate how the presented method can be used to study the dynamics of a system with shearing boundary conditions with a soft capsule adsorbed onto a fluid-fluid in the center of the system, where the shear is applied parallel to the interface. We initialize the system domain with 180 fluid nodes in both directions parallel to the fluid-fluid interface and 87 fluid sites perpendicular, where the odd numbered system height is chosen in order to easily initialize the interface exactly halfway between the shearing boundaries. To disable fluid-fluid interactions between the top and bottom planes, these are initialized with nonmoving bounce-back boundaries. Onsite velocity boundary conditions are applied on the two planes of fluid nodes next to these boundaries to impose the shear velocities [64,65].

When no shear is applied, i.e., $\mathrm{Ca}=0$, the particle has two symmetric major semiaxes parallel to the fluid-fluid interface and one minor semiaxis perpendicular to the interface, similar as previously visualized in Fig. 6. In the top panel of Fig. 10 we show the steady-state shape of the particle and the interface (a)

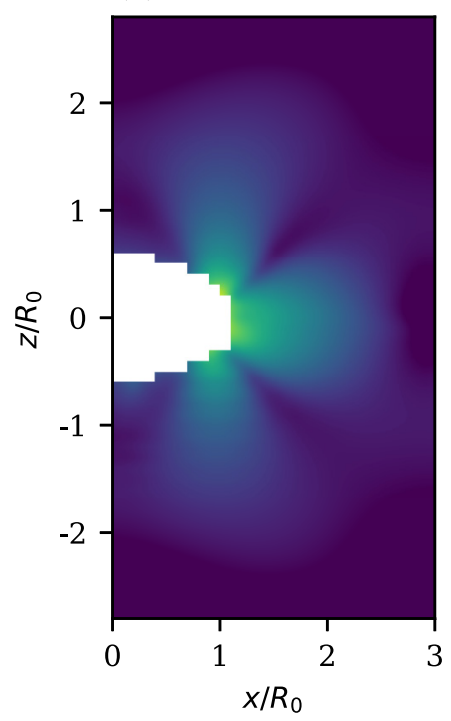

(b)

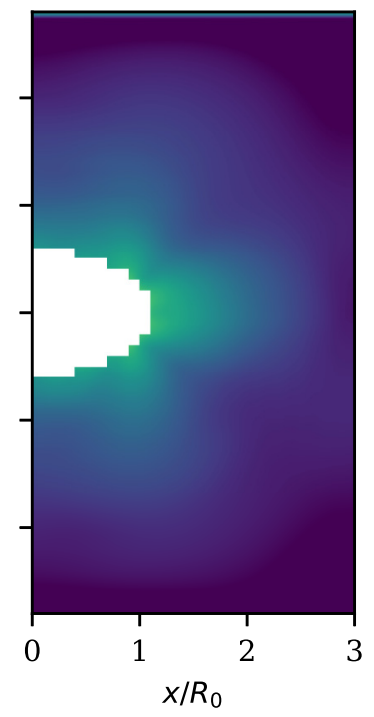

Magnitude of spurious velocities $[\Delta x / \Delta t]$

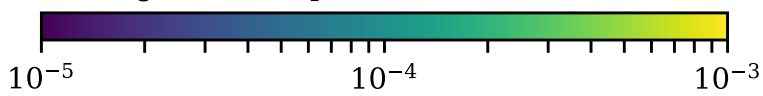

FIG. 9. Spurious velocities near the contact point of a soft particle with a fluid-fluid interface. Due to the curved boundary, the pseudopotential multicomponent fluid method will always yield spurious velocities near the contact point with the fluid interface. The velocity field outside the particle boundary is visualized on a vertical slice through the center of the particle, where the fluid nodes encapsulated by the boundary are shown in white. (a) Magnitude of the spurious velocities around a stationary soft particle with $\beta=500$ and $v_{s}=0.9$. (b) Magnitude of the spurious velocities around a particle comoving with the reference frame at a velocity of $\mathbf{v}=$ $(0.001,0.002,0.0)$. The reference frame is driven with a constant velocity $\mathbf{v}$ parallel to the fluid interface by using onsite velocity boundary conditions on the top and bottom planes. As a result of the changing discretization of the particle boundary on the fluid lattice, the averaged spurious velocities are smeared out.

for different capillary numbers and softness parameters. Here, it can be observed that the symmetry is broken when the system is sheared, and the major semiaxis parallel to the shear flow elongates. In the bottom panel of Fig. 10 we visualize the ratio between these two axes (the solid lines and symbols), and it can be clearly seen that the asymmetry for the two largest axes increases for increasing capillary numbers and decreasing particle softness. Simultaneously, the ratio between the major and minor axis deviates stronger from the base curve set by $\mathrm{Ca}=0.0$ for lower softness parameters when the capillary number is increased, while for large softness parameters the ratio becomes independent of the capillary number. Intuitively, this can be explained by the fact that softer particles have less area perpendicular to the shear flow, and therefore experience a lower stress from the shear flow. This results in a lower tank-treading velocity of the membrane, which is indeed observed in the simulations (data not shown).

In Fig. 11 we show the angle $\phi$ between the largest semiaxis and the horizontal plane (i.e., the undisturbed 

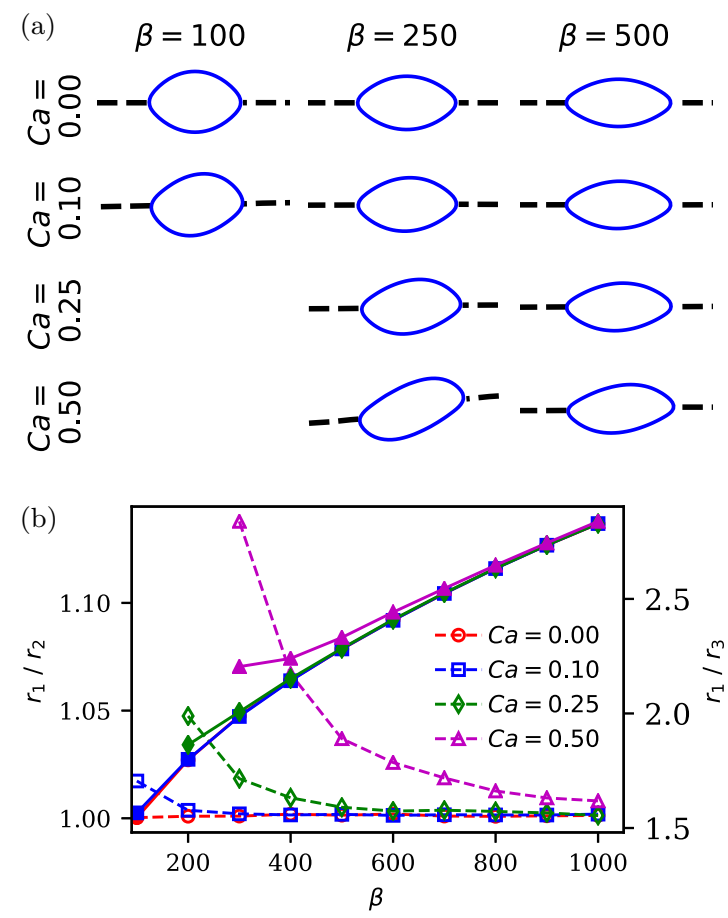

FIG. 10. Deformation of a soft particle adsorbed onto a fluidfluid interface with a shear velocity applied parallel to the interface. (a) Steady-state particle shapes (blue solid line) and fluid-fluid interface (black dashed line) for different capillary numbers and softness parameters, visualized as a 2D slice through the center of the particle. The fluid flows to the right at the top of the system, and to the left at the bottom of the system. For increasing capillary numbers, the asymmetry in the particle shape increases, where for the lower softness parameters the interface starts to deform as a result of the dynamical wetting of the tank-treading particle boundary. (b) Ratios between the semiaxes $r_{1}$ and $r_{2}$ (dashed lines and open markers) and $r_{1}$ and $r_{3}$ (solid lines and symbols) for different capillary numbers and softness parameters. The semiaxes are labeled in descending order labeled as $r_{n}$. For increasing softness parameters it can be observed that the influence of the shear flow reduces, and the ratio between $r_{1}$ and $r_{3}$ converges to the symmetric case of $\mathrm{Ca}=0.0$. The last shapes for $\beta=100$ and $\mathrm{Ca}=[0.25,0.5]$ are not included since the needed shear velocities approach the numerical limits of the LBM.

fluid-fluid interface), which increases for increasing capillary numbers and decreasing softness parameters. This rotation of the inertial tensor semiaxes and the corresponding asymmetry in the particle shape also influences the fluid-fluid interface, as can be seen in Fig. 10 for $\mathrm{Ca}=0.5$ and $\beta=250$. Here, one can observe the dynamical wetting of the particle surface, where the interface rises on one side of the particle while lowering on the other side in a symmetric manner due to the rotational velocity of the particle surface. Qualitatively, this fits with the expectations from experiments and simulations on similar systems with rigid particles [72], where the additional asymmetric deformation of the particle appears to allow a stronger deformation of the interface. A more detailed and thorough analysis of this phenomenon, including the effect of the preferential contact angle of the particle boundary, is left for future work.

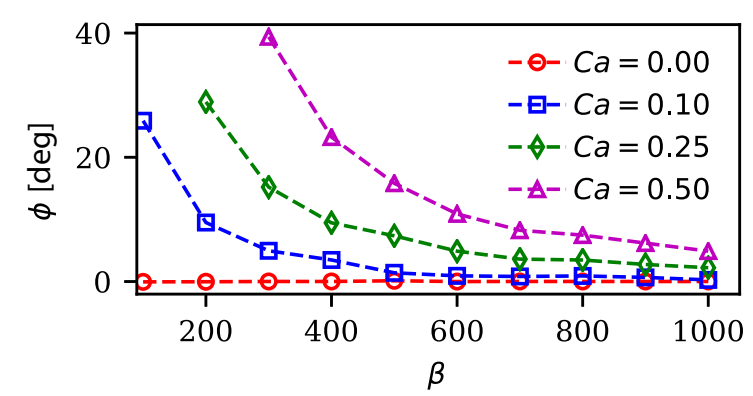

FIG. 11. Angle of largest semi-axis with the plane of the undisturbed interface for a soft particle adsorbed onto a fluid-fluid interface with a shear velocity applied parallel to the interface for different capillary numbers and softness parameters. For increasing capillary numbers the symmetry of the particle is broken as shown in Fig. 10, and the angle between the major axes and the horizontal plane increases.

\section{Tunable contact angles}

Similar to the work of Jansen et al. [29], our model is capable of tuning the preferential contact angle $\theta^{\text {eq }}$ of the particle surface with the fluid-fluid interface. A positive particle color $\Delta \rho$ is expected to result in the particle preferring one phase over the other phase, and a change from the $\theta^{\text {eq }}=90^{\circ}$ contact angle. For a rigid sphere with radius $R_{0}$ the contact angle can be measured by the distance $\delta z$ between the center of mass of the particle and the position of the interface as

$$
\cos \theta=\frac{\delta z}{R_{0}} .
$$

The particle is initially submerged in its preferred fluid component with the particle surface penetrating the fluid-fluid interface by roughly $1 \Delta x$. A rapid movement toward the equilibrium position, with some minor oscillations as a result of the inertia of the particle, can be observed as shown in Fig. 12.

From Young's equation the contact angle is given by the balance of the interfacial tensions $\gamma_{p c}$ and $\gamma_{p c^{\prime}}$ between the particle and two fluid components, and the tension $\gamma_{c c^{\prime}}$ between the two fluid components

$$
\cos \theta=\frac{\gamma_{p c}-\gamma_{p c^{\prime}}}{\gamma_{c c^{\prime}}}
$$

For a neutral particle color $\Delta \rho=0$ the fluid-structure tensions are symmetric and negate the nominator of Eq. (24). The virtual fluid density added to the fluid interaction force by a nonzero particle color increases the asymmetry in the two fluid-structure interfacial tensions and can be expected to yield a scaling of the equilibrium contact angle as

$$
\theta \propto \cos ^{-1}(\Delta \rho) \text {. }
$$

As seen in the right panel of Fig. 12, the measured equilibrium contact angle $\theta^{\text {eq }}$ of a rigid spherical boundary shows a good agreement with Eq. (25).

For rigid particles at a fluid-fluid interface, the vertical displacement $\delta_{z}=\left|z_{\text {interface }}-z_{\text {particle }}\right|$ is the only mechanism that allows for the match of the preferential contact angle $\theta^{\text {eq }}$ of the particle surface without deforming the interface. Soft particles, however, can also deform in order to match the 

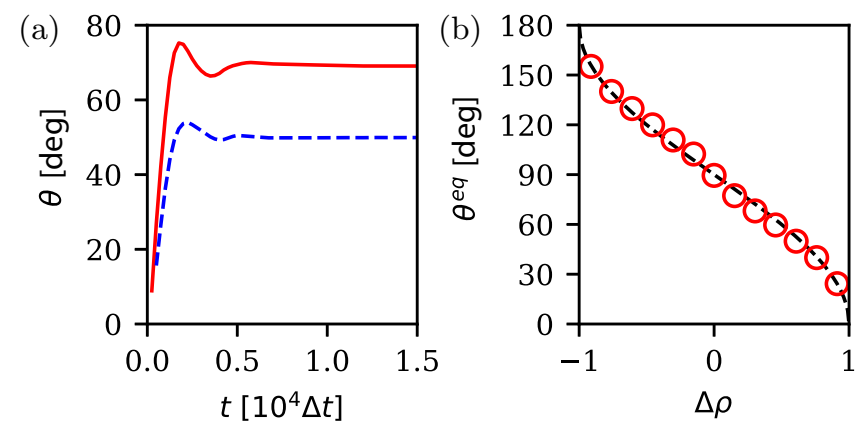

FIG. 12. Measured contact angle $\theta$ in degrees of rigid spherical boundaries with radius $R_{0}=10 \Delta x$. (a) Evolution of instantaneous contact angle as measured with Eq. (23) toward the equilibrium angle for a rigid capsule with a particle color of $\Delta \rho=0.30$ and 0.61 . To demonstrate the effect of the particle inertia, the particles are initialized inside their preferred fluid with the top of the particle touching the fluid-fluid interface. (b) Equilibrium contact angle as measured with Eq. (23) for different particle colors $\Delta \rho$ for rigid capsules. The dashed line gives the line $\theta=180 / \pi \cos ^{-1}(\Delta \rho)$, which shows good agreement with the obtained equilibrium contact angles from the simulation (red circles).

preferential contact angle. Thus, for larger values for the particle color, the equilibrium shape turns into a hazelnut shape, as depicted in the top panel of Fig. 13. This deformation simultaneously decreases the vertical displacement $\delta_{z}$ needed to match the preferential contact angle with the interface. In the bottom panel of Fig. 13 we clearly observe the influence of the particle softness $\beta$ on the displacement as compared to a rigid particle with an equal particle color $\Delta \rho$, where

(a)

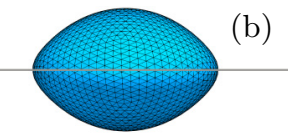

(d)

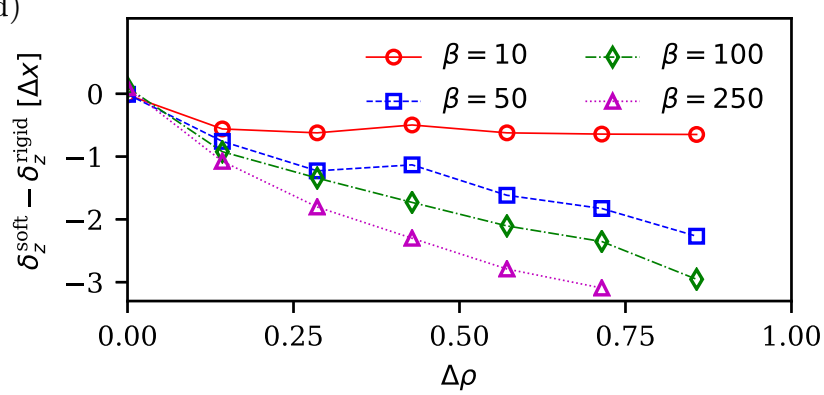

FIG. 13. Vertical displacement $\delta_{z}$ of the particle center of mass relative to the interface for increasing particle color $\Delta \rho$. (a)-(c) Equilibrium shapes of a soft capsule adsorbed onto an interface with $B=0.01, \beta=100, v_{s}=0.9$, and a particle color of, respectively, $\Delta \rho=[0.0,0.30,0.61]$ (from left to right). The fluid interface is shown by the gray line. (d) The difference in displacement of a soft particle $\delta_{z}^{\text {soft }}$ and a rigid particle $\delta_{z}^{\text {rigid }}$ for different softness parameters $\beta$. Soft particles are observed to penetrate less far into the preferred fluid as compared to rigid particles with the same preferred contact angle. For a very low softness $\beta=10$ the difference in displacement reaches a constant value particle color $\Delta \rho \geqslant 0.15$. (a)

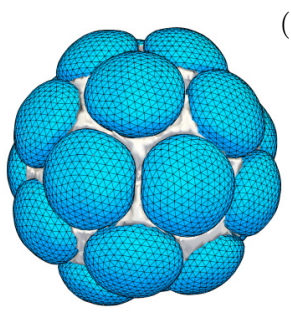

(b)

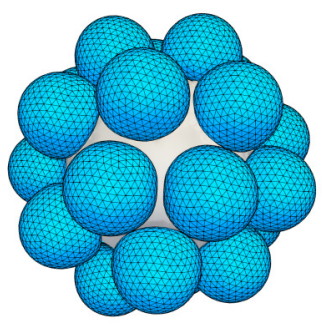

FIG. 14. Comparison of the equilibrium shapes of adsorbed soft particles at a droplet for particles with a particle color of (a) $\Delta \rho=$ 0.61 and (b) $\Delta \rho=-0.61$. The soft particles (blue) are initialized in a spherical configuration with $R=23 \Delta x$, centered around the droplet. All particles have an initial radius $R_{0}=7 \Delta x$, softness parameter $\beta=$ 100 , and Poisson ratio $v_{s}=0.9$. The droplet with an initial radius of $R=17.5 \Delta x$ is visualized with the isodensity line $\rho^{\text {drop }}=0.5$ (light gray), while the suspending bulk fluid is not visualized.

the particle displacement $\delta_{z}$ reduces for increasing softness parameters. For $\beta=100$ and $\Delta \rho=0.91$ the difference in displacement is almost $30 \%$ of the particle radius, while for relatively stiff capsules with $\beta=10$ the change in vertical displacement is only observed for low particle color values and reaches a constant difference for $\Delta \rho>0.15$.

\section{E. A droplet covered with soft particles}

To further demonstrate the capability of our method to simulate the interplay between soft particles and fluid-fluid interfaces, we simulate the covering of a droplet of radius $17.5 \Delta x$ suspended in another fluid by 24 soft capsules with $\beta=100$, $v_{s}=0.9$, and $R_{0}=7 \Delta x$. The particle centers are initialized on a sphere with radius $R=23 \Delta x$, such that each particle slightly penetrates the droplet. Two different simulations are shown, one with hydrophilic particles with $\Delta \rho=-0.61$ and another with hydrophobic particles with $\Delta \rho=0.61$. A short range repulsive hard-core particle-particle interaction force is added between boundary nodes of opposing particles to prevent the overlap of the particles

$$
F^{\mathrm{P}-\mathrm{P}}(d)= \begin{cases}\epsilon^{\mathrm{P}-\mathrm{P}}\left(d_{0}-d\right)^{3 / 2} & d<d_{0}, \\ 0 & d \geqslant d_{0},\end{cases}
$$

where $\epsilon^{\mathrm{P}-\mathrm{P}}$ is a constant parameter fixed here to $0.5, d$ is the separation distance between the two boundary nodes, and $d_{0}=1.25 \Delta x$ is a cutoff distance for the interaction force. The exact shape of the repulsive force is, however, unimportant as long as it is sufficiently strong to prevent the overlap of the particles.

In Fig. 14 we compare the obtained configurations of both simulations after $5 \times 10^{3} \Delta t$. The hydrophilic particles are almost completely pulled inside the droplet, covering nearly the entire surface of the droplet. Due to the close packing at the interface, the hydrophilic particles cannot obtain the preferred hazelnut shape as shown in Fig. 13, and the deformation is also significantly influenced by the interactions with other particles. The hydrophobic particles only slightly penetrate the surface of the droplet, resulting in a more compact droplet and a larger surface area uncovered by particles. A detailed investigation of this system goes beyond the scope of this work and will be presented in a future paper. 


\section{CONCLUSION AND DISCUSSION}

We have presented a method capable of simulating soft particles in multicomponent fluids, where we combined the bounce-back fluid-structure coupling with the multicomponent fluid model of Shan and Chen. By using a specially designed reference model, the method has been shown to achieve an excellent agreement for the deformation of a Skalak capsule at a fluid-fluid interface, where the errors stemming from the coupling between the multicomponent fluid and the particle surface are well below the characteristic length scale of the discretized fluid lattice. Despite the diffuse interface of the used multicomponent model, a good agreement is still observed for the equilibrium shapes of particles with radii comparable to the diffuse interface width.

The used fluid-structure coupling furthermore allows one to easily tune the contact angle of a single particle over a wide range of contact angles. It has been shown that the softness of the particle has a significant influence on the vertical displacement of the center of mass of the particle relative to the fluid interface, resulting from the additional deformation of the particle.

All individual simulations shown in this work can be comfortably run on a modern desktop, leaving ample room to increase the system size and number of modeled particles when using modern high-performance clusters. The implemented code has been successfully tested for large systems with up to 2000 particles on available high-performance computing clusters, and therefore paves the way to investigate a variety of systems where both fluid interfaces and the softness of suspended particles play a significant role.

There are also multiple opportunities to straightforwardly increase the level of complexity in the modeled systems within the presented method. For example, one can trivially move into the direction of inhomogeneous wetting particle surfaces, i.e., soft Janus particles, by defining the particle color individually on each boundary element. Furthermore, the bounce-back coupling can easily be altered to allow for a small permeability of the boundary by applying the bounceback coupling only to a fraction of the distribution functions, allowing to model the slow release of the interior fluid of the soft capsules.
The halfway bounce-back coupling introduces the wellknown staircasing effect in the discretization of the particle surface on the fluid lattice, thereby adding small discretization errors in the normal vector of the particle surface, which in turn result in small deviations of the expected equilibrium position. Furthermore, the bounce-back conditions introduce small force fluctuations in the force acting on the surface when the discretization on the fluid lattice changes (not shown in this paper). If desired, these effects can be mitigated by switching to higher-order coupling schemes, such as interpolated bounce-back, but one needs to keep in mind that such methods break the local mass conservation. During the development of the present method, the small mass errors from interpolated bounce-back schemes have been observed to quickly result in a drift of the mass in the system since the density gradients close to the fluid interface are a priori large. In contrast to the mass change due to the fresh node interpolation, which was remedied by using the adaptive rescaling proposed by Jansen et al., the mass change from interpolated bounce-back also occurs for particles which are fixed in space and time. The used mass-correction term is unsuitable to correct for these errors, and devising another mass-conserving scheme without significantly afflicting the local fluid velocity is nontrivial. Hence, for the applications of dense suspensions the halfway bounce-back boundary condition is the most suitable since it only requires a single fluid node between two adjacent particles, whereas the higher-order methods typically require two or three fluid nodes.

\section{ACKNOWLEDGMENTS}

Special thanks go out to M. Zellhöfer for help with various technical aspects during the code development. We acknowledge NWO/TTW (Project No. 10018605), HPC-Europa3 (Grant No. INFRAIA-2016-1-730897), and the DFG within the Cluster of Excellence "Engineering of Advanced Materials" (Project No. EXC315, Bridge funding) for available funding, and the Jülich Supercomputing Centre and the High Performance Computing Centre Stuttgart for the allocated CPU time. T.K. thanks the University of Edinburgh for support through a Chancellor's Fellowship.
[1] P. P. Constantinides, Lipid microemulsions for improving drug dissolution and oral absorption: Physical and biopharmaceutical aspects, Pharm. Res. 12, 1561 (1995).

[2] M. Y. Levy and S. Benita, Drug release from submicronized o/w emulsion: A new in vitro kinetic evaluation model, Int. J. Pharm. 66, 29 (1990).

[3] C. Puglia and F. Bonina, Lipid nanoparticles as novel delivery systems for cosmetics and dermal pharmaceuticals, Expert Opin. Drug Delivery 9, 429 (2012).

[4] H. D. Silva, M. Â. Cerqueira, and A. A. Vicente, Nanoemulsions for food applications: Development and characterization, Food Bioprocess Technol. 5, 854 (2012).

[5] J. Keddie and A. Routh, Fundamentals of Latex Film Formation: Processes and Properties (Springer, Berlin, 2010).
[6] H. van der Kooij, G. van de Kerkhof, and J. Sprakel, A mechanistic view of drying suspension droplets, Soft Matter 12, 2858 (2016).

[7] T. Krüger, Effect of tube diameter and capillary number on platelet margination and near-wall dynamics, Rheol. Acta 55, 511 (2016).

[8] M. Mehrabadi, D. N. Ku, and C. K. Aidun, Effects of shear rate, confinement, and particle parameters on margination in blood flow, Phys. Rev. E 93, 023109 (2016).

[9] M. Gross, T. Krüger, and F. Varnik, Rheology of dense suspensions of elastic capsules: Normal stresses, yield stress, jamming and confinement effects, Soft Matter 10, 4360 (2014).

[10] T. Krüger, B. Kaoui, and J. Harting, Interplay of inertia and deformability on rheological properties of a suspension of capsules, J. Fluid Mech. 751, 725 (2014). 
[11] R. M. MacMeccan, J. R. Clausen, G. P. Neitzel, and C. K. Aidun, Simulating deformable particle suspensions using a coupled lattice-Boltzmann and finite-element method, J. Fluid Mech. 618, 13 (2009).

[12] S. Frijters, F. Günther, and J. Harting, Effects of nanoparticles and surfactant on droplets in shear flow, Soft Matter 8, 6542 (2012).

[13] F. Günther, S. Frijters, and J. Harting, Timescales of emulsion formation caused by anisotropic particles, Soft Matter 10, 4977 (2014).

[14] H. Mehrabian, J. Harting, and J. Snoeijer, Soft particles at a fluid interface, Soft Matter 12, 1062 (2016).

[15] O. Zienkiewicz, R. Taylor, and P. Nithiarasu, The Finite Element Method for Fluid Dynamics, 7th ed. (Butterworth-Heinemann, Oxford, 2014).

[16] C. K. Aidun and C. J. Clausen, Lattice-Boltzmann method for complex flows, Annu. Rev. Fluid Mech. 42, 439 (2010).

[17] J. Harting, M. Venturoli, and P. V. Coveney, Large-scale gridenabled lattice Boltzmann simulations of complex fluid flow in porous media and under shear, Philos. Trans. R. Soc. A 362, 1703 (2004).

[18] A. J. C. Ladd and R. Verberg, Lattice-Boltzmann simulations of particle-fluid suspensions, J. Stat. Phys. 104, 1191 (2001).

[19] C. Bächer, L. Schrack, and S. Gekle, Clustering of microscopic particles in constricted blood flow, Phys. Rev. Fluids 2, 013102 (2017).

[20] T. Krüger, F. Varnik, and T. Raabe, Efficient and accurate simulations of deformable particles immersed in a fluid using a combined immersed boundary lattice Boltzmann finite element method, Comput. Math. Appl. 61, 3485 (2011).

[21] A. Farutin, Z. Shen, G. Prado, V. Audemar, H. Ez-Zahraouy, A. Benyoussef, B. Polack, J. Harting, P. M. Vlahovska, T. Podgorski, G. Coupier, and C. Misbah, Optimal cell transport in straight channels and networks, Phys. Rev. Fluids 3, 103603 (2018).

[22] I. Halliday, S. V. Lishchuk, T. J. Spencer, G. Pontrelli, and P. C. Evans, Local membrane length conservation in twodimensional vesicle simulation using multi-component lattice Boltzmann equation method, Phys. Rev. E 94, 023306 (2016).

[23] B. Kaoui, J. Harting, and C. Misbah, Two-dimensional vesicle dynamics under shear flow: Effect of confinement, Phys. Rev. E 83, 066319 (2011).

[24] G. A. Buxton, C. M. Care, and D. J. Cleaver, A lattice spring model of heterogeneous materials with plasticity, Modell. Simul. Mater. Sci. Eng. 9, 485 (2001).

[25] J. Wu and C. K. Aidun, Simulating 3D deformable particle suspensions using lattice Boltzmann method with discrete external boundary force, Int. J. Numer. Methods Fluids 62, 765 (2010).

[26] A. J. C. Ladd, Numerical simulations of particulate suspensions via a discretized Boltzmann equation. Part 1: Theoretical foundation, J. Fluid Mech. 271, 285 (1994).

[27] M. Cheng, B. Zhang, and J. Lou, A hybrid LBM for flow with particles and drops, Comput. Fluids 155, 62 (2017).

[28] S. Frijters, F. Günther, and J. Harting, Domain and droplet sizes in emulsions stabilized by colloidal particles, Phys. Rev. E 90, 042307 (2014).

[29] F. Jansen and J. Harting, From bijels to Pickering emulsions: A lattice Boltzmann study, Phys. Rev. E 83, 046707 (2011).

[30] A. S. Joshi and Y. Sun, Multiphase lattice Boltzmann method for particle suspensions, Phys. Rev. E 79, 066703 (2009).
[31] K. W. Connington, T. Lee, and J. F. Morris, Interaction of fluid interfaces with immersed solid particles using the lattice Boltzmann method for liquid-gas-particle systems, J. Comput. Phys. 283, 453 (2015).

[32] K. Stratford, R. Adhikari, I. Pagonabarraga, and J. C. Desplat, Lattice Boltzmann for binary fluids with suspended colloids, J. Stat. Phys. 121, 163 (2005).

[33] H. Tanaka and T. Araki, Simulation Method of Colloidal Suspensions with Hydrodynamic Interactions: Fluid Particle Dynamics, Phys. Rev. Lett. 85, 1338 (2000).

[34] Z.-G. Feng and E. E. Michaelides, The immersed boundarylattice Boltzmann method for solving fluid-particles interaction problems, J. Comput. Phys. 195, 602 (2004).

[35] J. Wu and C. Shu, Implicit velocity correction-based immersed boundary-lattice Boltzmann method and its applications, J. Comput. Phys. 228, 1963 (2009).

[36] Z. Li, J. Favier, U. D’Ortona, and S. Poncet, An immersed boundary-lattice Boltzmann method for single- and multicompoent fluid flows, J. Comput. Phys. 304, 424 (2016).

[37] X. Shan and H. Chen, Lattice Boltzmann model for simulating flows with multiple phases and components, Phys. Rev. E 47, 1815 (1993).

[38] X. Shan and G. Doolen, Multicomponent lattice-Boltzmann model with interparticle interaction, J. Stat. Phys. 81, 379 (1995).

[39] C. S. Peskin, Flow patterns around heart valves: A numerical method, J. Comput. Phys. 10, 252 (1972).

[40] M. Pepona, A. C. M. Shek, C. Semprebon, T. Krüger, and H. Kusumaatmaja, Modelling ternary fluids in contact with elastic membranes, arXiv:1906.03065.

[41] A. Pinelli, I. Z. Naqavi, U. Piomelli, and J. Favier, Immersedboundary methods for general finite-difference and finitevolume Navier-Stokes solvers, J. Comput. Phys. 229, 9073 (2010).

[42] D. A. Reasor, J. R. Clausen, and C. K. Aidun, Coupling the lattice-Boltzmann and spectrin-link methods for the direct numerical simulation of cellular blood flow, Int. J. Numer. Methods Fluids 68, 767 (2011).

[43] R. Skalak, A. Tozeren, R. P. Zarda, and S. Chien, Strain energy function of red blood cell membranes, Biophys. J. 13, 245 (1973).

[44] D. Barthés-Biesel, Motion and deformation of elastic capsules and vesicles in flow, Annu. Rev. Fluid Mech. 48, 25 (2016).

[45] T. Biben, A. Farutin, and C. Misbah, Three-dimensinoal vesicles under shear flow: Numerical study of dynamics and phase diagram, Phys. Rev. E 83, 031921 (2011).

[46] R. Benzi, S. Succi, and M. Vergassola, The lattice Boltzmann equation: Theory and applications, Phys. Rep. 222, 145 (1992).

[47] T. Krüger, H. Kusumaatmaja, A. Kuzmin, O. Shardt, G. Silve, and E. M. Viggen, The Lattice Boltzmann Method: Principles and Practice, Graduate Texts in Physics (Springer, Berlin, 2017).

[48] S. Succi, The Lattice Boltzmann Equation: For Fluid Dynamics and Beyond, Numerical Mathematics and Scientific Computation (Clarendon, Oxford, 2001).

[49] P. L. Bhatnagar, E. P. Gross, and M. Krook, A model for collision processes in gases. I. Small amplitude processes in charged and neutral one-component systems, Phys. Rev. 94, 511 (1954). 
[50] J. M. Charrier, S. Shrivastava, and R. Wu, Free and constrained inflation of elastic membranes in relation to thermoformingnon-axisymmetric problems, J. Strain Anal. Eng. Des. 24, 55 (1989).

[51] S. Shrivastava and J. Tang, Large deformation finite element analysis of non-linear viscoelastic membranes with reference to thermoforming, J. Strain Anal. Eng. Des. 28, 31 (1993).

[52] W. Helfrich, Elastic properties of lipid bilayers: Theory and possible experiments, Z. Naturforsch. Teil C 28, 693 (1973).

[53] M. Meyer, M. Desbrun, P. Schröder, and A. H. Barr, Discrete differential-geometry operators for triangulated 2-manifolds, in Visualization and Mathematics III, edited by H.-C. Hege and K. Polthier (Springer, Berlin, 2003), pp. 35-57.

[54] D. Barthés-Biesel, A. Diaz, and E. Dhenin, Effect of constitutive laws for two-dimensional membranes on flow-induced capsule deformation, J. Fluid Mech. 460, 211 (2002).

[55] J. R. Clausen and C. K. Aidun, Capsule dynamics and rheology in shear flow: Particle pressure and normal stress, Phys. Fluids 22, 123302 (2010).

[56] G. A. Buxton, R. Verberg, D. Jasnow, and A. C. Balazs, Newtonian fluid meets an elastic solid: Coupling lattice Boltzmann and lattice-spring models, Phys. Rev. E 71, 056707 (2005).

[57] I. Ginzbourg and D. d'Humières, Local second-order boundary methods for lattice Boltzmann models, J. Stat. Phys. 84, 927 (1996).

[58] S. Khirevich, I. Ginzburg, and U. Tallarek, Coarse- and fine-grid numerical behavior of MRT/TRT lattice-Boltzmann schemes in regular and random sphere packings, J. Comput. Phys. 281, 708 (2015).

[59] M. C. Sukop and D. T. Thorne, Lattice Boltzmann Modeling: An Introduction for Geoscientists and Engineers (Springer, Berlin, 2006).

[60] M. Bouzidi, M. Firdaouss, and P. Lallemand, Momentum transfer of a Boltzmann-lattice fluid with boundaries, Phys. Fluids 13, 3452 (2001).

[61] I. Ginzburg and D. d'Humiéres, Multireflection boundary conditions for lattice Boltzmann models, Phys. Rev. E 68, 066614 (2003).
[62] S. Tao, Q. He, B. Chen, X. Yang, and S. Huang, One-point second-order curved boundary condition for lattice Boltzmann simulation of suspended particles, Comput. Math. Appl. 76, 1593 (2018).

[63] C. K. Aidun, Y. Lu, and E.-J. Ding, Direct analysis of particulate suspensions with inertia using the discrete Boltzmann equation, J. Fluid Mech. 373, 287 (1998).

[64] Q. Zou and X. He, On pressure and velocity boundary conditions for the lattice Boltzmann BGK model, Phys. Fluids 9, 1591 (1997).

[65] M. Hecht and J. Harting, Implementation of on-site velocity boundary conditions for D3Q19 lattice Boltzmann, J. Stat. Mech.: Theory Exp. (2010) P01018.

[66] S. Ramanujan and C. Pozrikidis, Deformation of liquid capsules enclosed by elastic membranes in simple shear flow: Large deformations and the effect of fluid viscosities, J. Fluid Mech. 361, 117 (1998).

[67] E. Lac and D. Barthés-Biesel, Deformation of a capsule in simple shear flow: Effect of membrane prestress, Phys. Fluids 17, 072105 (2005).

[68] P. Bagchi and R. M. Kalluri, Rheology of a dilute suspension of liquid-filled elastic capsules, Phys. Rev. E 81, 056320 (2010).

[69] T. Omori, T. Ishikawa, D. Barthés-Biesel, A.-V. Salsac, J. Walter, Y. Imai, and T. Yamaguchi, Comparison between spring network models and continuum constitutive laws: Application to the large deformation of a capsule in shear flow, Phys. Rev. E 83, 041918 (2011).

[70] A. Guckenberger and S. Gekle, Theory and algorithms to compute Helfrich bending forces: A review, J. Phys.: Condens. Matter 29, 203001 (2017).

[71] K. Tsubota, Short note on the bending models for a membrane in capsule mechanics: Comparison between continuum and discrete models, J. Comput. Phys. 277, 320 (2014).

[72] S. Cappelli, Q. Xie, J. Harting, A. M. de Jong, and M. W. J. Prins, Dynamic wetting: Status and prospective of single particle based experiments and simulations, New Biothec. 32, 420 (2015). 